\title{
COVID-19 Importation Risk From Olympic Athletes Prior to the Tokyo 2020 Olympics
}

\begin{abstract}
Hoi Yat Vico Lau ${ }^{1,2}$, Mingda X $u^{1,2}$, Lin Wang ${ }^{3}$, Benjamin J. Cowling ${ }^{1,2}$ and Zhanwei Du ${ }^{1,2 *}$
${ }^{1}$ WHO Collaborating Centre for Infectious Disease Epidemiology and Control, School of Public Health, Li Ka Shing Faculty of Medicine, The University of Hong Kong, Hong Kong, Hong Kong, SAR China, ${ }^{2}$ Laboratory of Data Discovery for Health, Hong Kong Science and Technology Park, Hong Kong, Hong Kong, SAR China, ${ }^{3}$ Department of Genetics, University of Cambridge, Cambridge, United Kingdom
\end{abstract}

The COVID-19 pandemic delayed the Tokyo 2020 Olympics for 1 year and sparked an unprecedented outbreak in Japan in early July 2021 due to the relaxation of social distancing measures for foreign arrivals. Approximately 11,000 athletes from 205 countries would gather at the Tokyo Olympics held from July 23 through August 8, 2021. Based on the prevalence of infection in different source locations and athlete numbers, we estimated that seven countries would introduce least one infection of COVID19 to Tokyo and at most eleven unidentified infections after the three requested COVID19 tests.

Edited by:

Hui-Jia Li,

Beijing University of Posts and Telecommunications (BUPT), China

Reviewed by: Dong Li,

Shandong University, China

Chijun Zhang,

Jilin University of Finance and

Economics, China

*Correspondence:

Zhanwei Du

zwdu@hku.hk

\section{Specialty section: \\ This article was submitted to Social Physics, a section of the journal Frontiers in Physics}

Received: 12 August 2021 Accepted: 01 September 2021 Published: 07 October 2021

Citation:

Vico Lau HY, Xu M, Wang L, Cowling BJ and DuZ (2021) COVID-19 Importation Risk From Olympic Athletes Prior to the Tokyo 2020 Olympics.

Front. Phys. 9:757361. doi: 10.3389/fphy.2021.757361
Keywords: Coronavirus, importation, infections, prevention, testing

\section{INTRODUCTION}

The risk of overwhelming fragile public health systems still lies in the coronavirus disorder 2019 (COVID-19), especially in developing countries. The COVID-19 in Japan rose sharply in early July 2021. As of August 22, 2021, there are 1.28 million COVID-19 confirmed cases reported, coupled with 16 thousand deaths. Globally, there have been over 211 million reported cases and 4.42 million deaths [1].

In September 2013, Tokyo in Japan was awarded the privilege to host the 2020 Olympics for the second time [2]. Hosting the Olympics has held great importance for Japan as the 1964 Games were seen as a symbol of revival for the country's rehabilitation and rebuilding process after World War Two, especially when Japan is currently facing a prolonged economic stagnation and recession [3]. However, the World Health Organization (WHO) declared the Coronavirus outbreak as a global public health emergency in January 2020 only months before the Olympics were scheduled to be held, this pressured the International Olympic Committee (IOC) to postpone the Games by a year with a cost of approximately $\$ 2.8$ billion USD [2]. As of January 2021, Japan's government decided to continue this event amid the pandemic to avoid $\$ 15.4$ billion USD in loss if canceled $[2,4]$.

In May 2021, the Tokyo Medical Practitioners Association, as well as Japanese firms, called to cancel the Games with the fear of facing another wave of infections [2]. This fear quickly turned to reality as Japan faced the fourth wave of infections in mid-June 2021 and outbreaks appeared in several cities in Japan, including the host city, Tokyo [5]. The Tokyo Olympics has taken measures to prevent the spread of the virus. In July 2021, IOC announced that there will be no spectators, which would see $\$ 820$ million USD in lost revenue, to mitigate the potential outbreak [6]. But as to how specific and efficient the policies are still questionable. Athletes are required to undergo three COVID-19 tests before entering the Olympic Village, two of which should be done within $96 \mathrm{~h}$ of their flights to Tokyo and one test done on arrival [7]. However, COVID-19 tests can be negative 
during the latent period, and may not be able to identify all the infected athletes and prevent all introductions of infection into the Olympic village and potentially the local community.

Over 11,000 athletes from 205 countries would gather at the Tokyo Olympics held from July 23 through August 8, 2021 [8]. To build a COVID-19 bubble isolated from the Japanese population, there have been measures tailored towards preventing the spread of the virus. However, some measures were criticized by experts as they believe that the "safety protocols do not adequately protect athletes" and the "measures may not be strong enough to prevent outbreaks" [9]. The facilities in place were not designed to mitigate the risks that the pandemic might pose. The control measures and facilities provided could potentially be insufficient to prevent an outbreak from happening inside the COVID-19 bubble in the Olympic village. To quantify the transportation risk of athletes from each of the 205 other countries prior to the Tokyo 2020 Olympics, we would evaluate the probability of at least one infection and the number of infections arriving in Tokyo even after the three requested tests among athletes prior to the Tokyo 2020 Olympics.

\section{MATERIALS AND METHODS}

\section{Risk of Imported Infections From Participating Countries to Tokyo}

We used the number of athletes who traveled into Tokyo and the infection rate per person from each of the participating countries during the period from July 1 to July 23, $2021[10,11]$, to calculate the probable number of imported infected cases from participating countries. The following equation is used to deduce the probability of infected athletes traveling into Tokyo from active cases in the country $i$ by

$$
\gamma_{i}=\alpha_{i} \beta_{i}
$$

Here $\alpha_{i}$ denotes the average probability of a person being infected in the country $i$, estimated bythe country's active cases per person. The number of active cases was the average active cases between the athletes' traveling period, July 1 to July 23, 2021. Then, it is multiplied by the number of participating athletes $\left(\beta_{i}\right)$ to evaluate the probable number of infected athletes that the country would import into Tokyo.

\section{Testing Strategy}

The IOC playbook for players has adopted a testing strategy where foreign players would take three COVID-19 tests and local players would take two [7]. Since the test would most likely be taken on three different days for foreign players and the probability of a missed infection differs from day to day postinfection, the probability of a missed infection is deduced with the probability of the day of the infection, the day post-infection, and 2 days post-infection. Whereas the probability of Japanese athletes would be calculated by the day of the infection and the next day. The test chosen is the antigen test as it is the test that athletes would most likely take and it is also the test that would be used on arrival. The antigen test is also the least effective between the eligible tests that could be taken which are molecular and antibody tests [12].

We can quantify the overall probable number of unidentified infections of all the countries combined after each COVID-19 test by accumulating the number of unidentified infections after each test from each country other than Tokyo. The probable number of unidentified infected athletes after testing $(\zeta)$ is given by

$$
\zeta=\gamma_{i} \prod_{j}\left(1-\epsilon_{j}\right)
$$

Here $\gamma_{i}$ denotes the probable number of imported infected athletes of the country $i . \epsilon_{j}$ represents the sensitivity of COVID19 tests with day $j$ post-infection.

\section{Alternative Strategy}

A more effective measure could be mandatory quarantine and testing. A successful example would be the NBA Bubble (July 12-October 11, 2020) where players were required to self-isolate in their rooms for up to $48 \mathrm{~h}$ until receiving two negative COVID19 tests with only two infections in the first week (July 22-28, 2020) and no infections after [13]. Inspired by this, we would estimate the probable number of unidentified COVID-19 cases if all athletes are self-isolated for more days after arriving. The probable number of unidentified cases would be given by

$$
\theta_{k}=\theta_{k-1} \cdot \iota_{k}
$$

Here $\theta_{k}$ represent the probable unidentified cases on the day $k$. $\iota k$ denotes the sensitivity of COVID-19 tests on day $k$

\section{RESULTS}

\section{Risk of Imported Infections From Participating Countries to Tokyo}

The risk for the transportation of COVID-19 to Tokyo varies across countries informed by the number of active cases during the studied period between July 1 to July 23, 2021 (Figure 1). The number of infected athletes arriving in Tokyo between July 1 to July 23, 2021, ranges from approximately two to six infections depending on the day of arrival. With limited outliers in the data, the average is taken to represent the probable number of infections that would be imported to Tokyo from Great Britain. Hence, the probable number of imported cases is 3.81 , so approximately four cases.

The United States and Great Britain have the highest probable cases with nine and four imported cases, respectively, followed by Spain, Russia, Netherlands, Brazil, and Argentina with at least one COVID-19 case into Tokyo. The estimated overall accumulated infections can be approximately rounded to 32 infected athletes in the studied period from July 1 to July 23, 2021. This would imply that the United States and Great Britain possess the highest risk of bringing multiple infected athletes into Tokyo along with Spain, Russia, Netherlands, Brazil, and Argentina.

\section{Testing Strategies}

We compare IOC's testing strategy in various days post-infection with an increase in sensitivity of the antigen COVID-19 test in the 
A

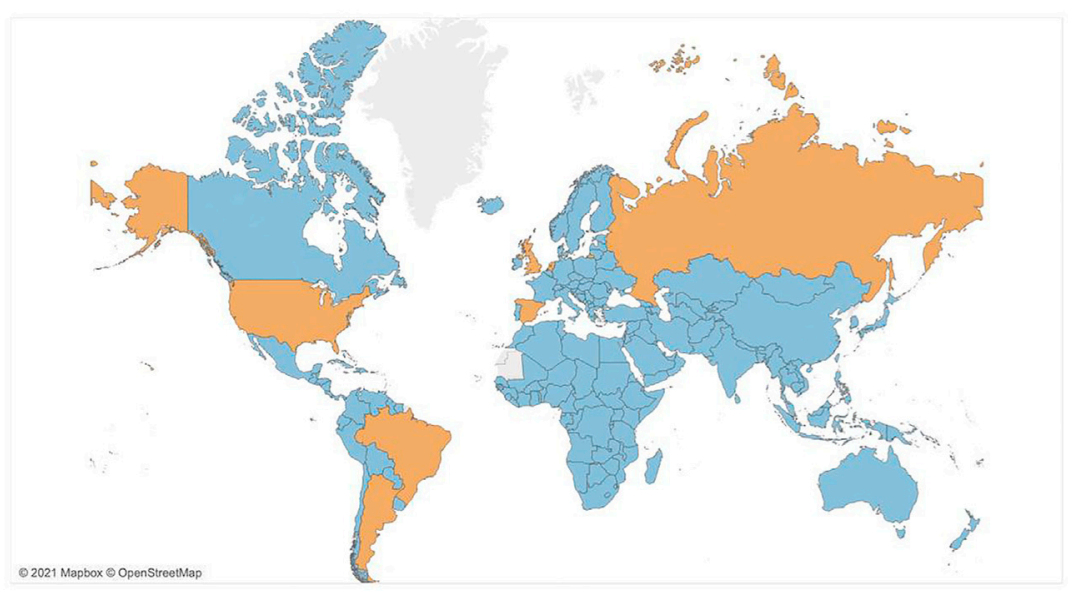

B

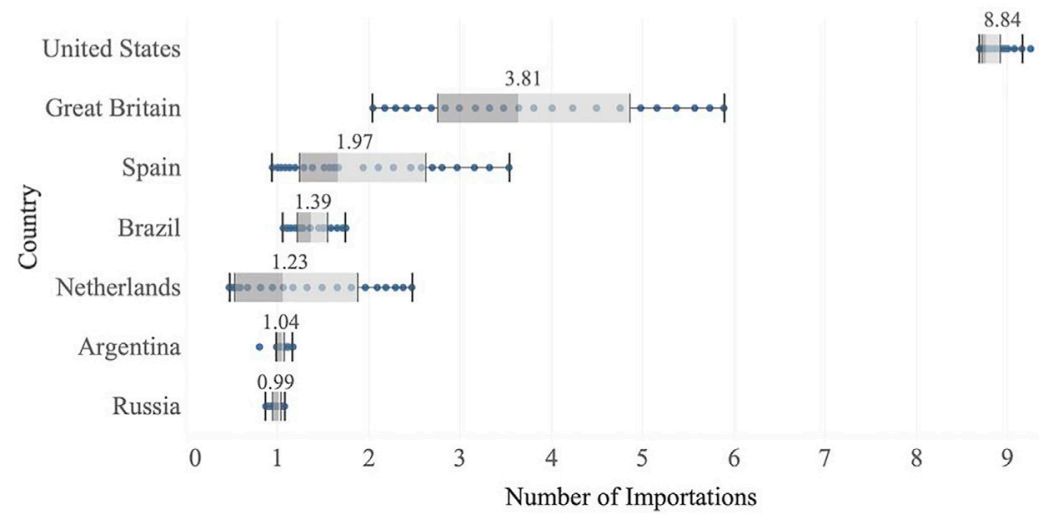

FIGURE 1 |Risks for transportation of COVID-19 to Tokyo, Japan, between July 1 to July 23, 2021. (A) Estimated median probable number of athletes imported to Tokyo from participating countries into Tokyo during the studied period between July 1 to July 23, 2021. For each country, we estimate probable numbers by assuming athletes all travel to Tokyo on a day during the studied period, resulting in 23 estimates. The seven countries with a median probable number $>1$ are indicated in shades of orange; 198 countries below that threshold are indicated in shades of blue. (B) Estimated probable numbers of athletes in seven countries with the largest median estimates of probable numbers. The box plots denote a five-number summary of minimum, lower quartile, median, upper, and maximum values based on the 23 estimated numbers. The labeled value above the box plot is the average number of importations. The map was created using Tableau Software for Desktop version 2021.2 (https://www.tableau.com/support/releases/desktop/2021.2). The layouts were modified with Keynote version 11.1 (https://www.apple.com/hk/en/keynote/).

following days (Figure 2A). The sensitivity of the antigen COVID-19 test ranges from 26.3 to $100 \%$ with a steady increase in sensitivity daily during the first 6 days postinfection [12]. Given there are 32 athletes estimated to be imported into Tokyo in Japan, we estimate the number of unidentified infections across three COVID-19 tests (Figure 2A). There would still be 11 unidentified cases after three antigen COVID-19 tests if the first test was taken coincidently on the day of infection. Specifically, the test on the first, second and third day post-infection has sensitivities of $26.3,30.0$, and $36.3 \%$, respectively, resulting in reduced importation from 32 cases to 24,17 , and 11 , respectively. In contrast, there would only be five unidentified infections finally if the first tests were all taken on the second day post-infection and can be zero if the first tests are all conducted 3 days or later postinfection.
We illustrate the decrease in the number of unidentified infections with the use of the increased sensitivity of the antigen COVID-19 test in the following days (Figure 2B). This shows that with daily testing for 5 days, the risk of an infected person is closer to zero than one with the day of infection on the day of the first test. The day of infection on the day of the first test was chosen to portray the most pessimistic assumption for COVID-19 testing since the test is least effective when the person is recently infected. Mandatory quarantine of the player in their rooms was chosen to avoid the risk of the virus transmitting through contact and interaction. We can imply that the risk of an infected person entering the Olympic Village after quarantine and daily testing is extremely low. With implementing a 5 days quarantine and daily testing for the entry of athletes, the risk of the virus entering the Olympic Village has been driven down significantly. 


\section{Testing Strategies}

A

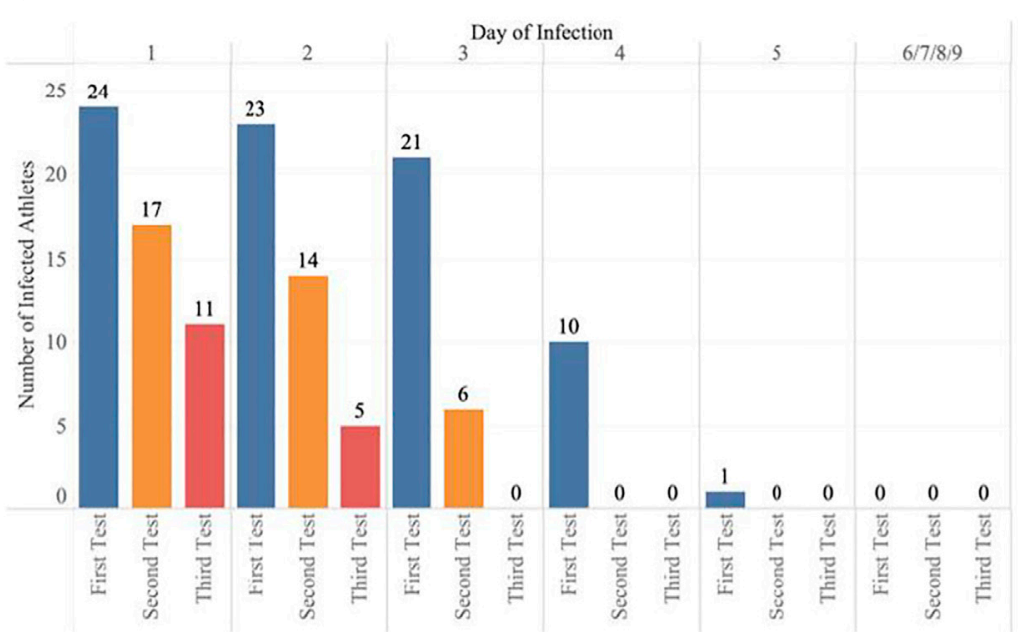

B

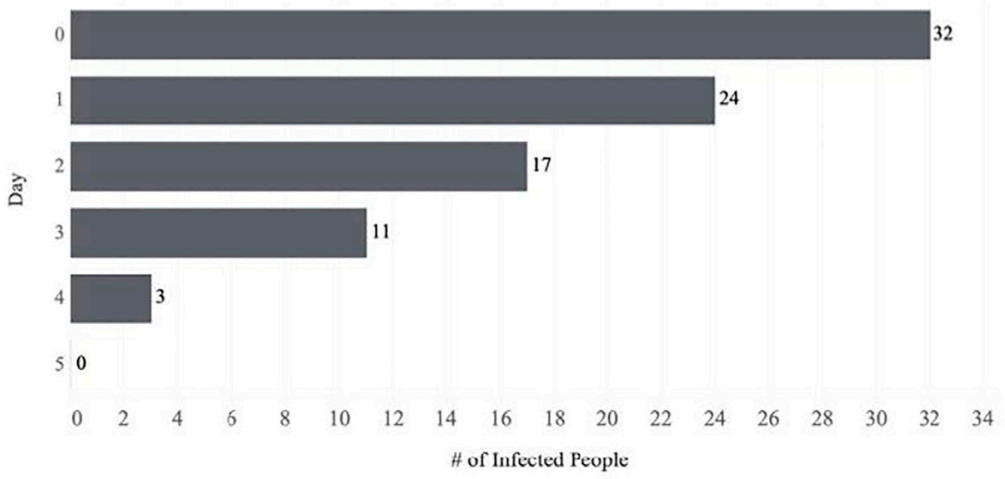

FIGURE 2 | The number of infected athletes after each test for the respective strategies. (A) The probable number of unidentified cases with the IOC's strategy by using the probable number of unidentified cases and the sensitivity of each test executed during post-infection [12]. We assume tests are taken on the first, second, and third day post-infection, which are colored by blue, orange, and red, respectively. This value in the $y$-axis infers the number of unidentified infected athletes after each test. (B) The number of infected athletes after 5 days of quarantine and regular testing. This figure extrapolates the idea of how the number of quarantine days decreases the chance of unidentified infections while regularly tested in isolation. The bar graph and line graph were created using Tableau Software for Desktop version 2021.2 (https://www.tableau.com/support/releases/desktop/2021.2). The layouts were modified with Keynote version 11.1 (https:// www.apple.com/hk/en/keynote/).

\section{DISCUSSION AND CONCLUSION}

Despite the efforts of the implementation of guidelines to contain the spread of the virus via the official playbook, the measures and facilities in place cannot prevent the burst of the coronavirus bubble. Even though vaccinated players have an increase in immunity, they can also be infected along with the unvaccinated players. Accurate testing before entry to the coronavirus bubble is essential as the virus would assuredly spread among athletes inside the bubble with its shared space and loosened coronavirus protocols while competing. The unaddressed aerosol fine particle transmission and the contact tracing strategies are not adequate to fully monitor and safely control the risk of transmissions. It would be advised to enforce more specific and effective measures before the continuation of the Games.

Are the facilities and measures within the Olympic Village sufficient to prevent the break of the bubble?

The Tokyo Olympics 2020 attempts to construct a coronavirus bubble with several measures in place to control the spread of the virus such as regular testing, contact tracing, and social distancing [7]. According to the official playbook within the bubble, there are policies to be followed, but without enough details [7].

For example, the playbook of Tokyo Olympics 2020 does not address specific guidelines to prevent the most important transmission mode, aerosol fine particle transmission [7]. The significant features of exposure to aerosol inhalation are the 
concentration of infectious particles in the air and the length of time spent in contact with those particles [14]. Organizers were confident that it is sufficient to keep the athletes' room ventilated, but the specifics as to how frequently the air within indoor spaces was refreshed and replaced every hour were not given in detail [15]. There is also a lack of information about the review, adaptation, and modification of HVAC systems, which would pose a potential risk of unmonitored aerosol transmissions in various spaces inside the coronavirus bubble.

Contact tracing mainly relies on smartphones rather than wearable technology [7]. As smartphones would not always be present with the athletes while they are competing, wearable technology would be more efficient and safe for close contact tracking [14]. The current policy in the playbook to track contacts is by surveying with questions over the phone (how long were they together, whether they were wearing a mask, etc.), [7]. This strategy is not feasible as the player's participation would be at stake if they are verified as close contact, therefore the player could simply fabricate their statement for the continuation of their participation. Wearable technology backed up by video monitoring for finding close contact could and should be implemented into contact tracing as another option.

Other issues may arise too. Though the Olympic Village is expected to be a bubble isolated from the Japanese public, there would still be inevitable contact with service providers such as cleaning, transport, COVID-19 testing, security, and catering outside the bubble [16]. As seen in Australia, bubbles are still vulnerable to infections despite strict hygiene practices [16].

\section{Limitation of the Predicted Outcome of International Olympic Committee's Testing Plan}

There is also a risk of athletes being infected during their travels to Tokyo between the three COVID-19 tests taken in their country. Once the case is not identified by the first two tests, the virus could be easily transmitted during the flight, a confined space. There is a high probability that the infection would be unidentified as they would only take one COVID-19 test on arrival. With no quarantine measures taken, the virus could potentially enter the Olympic Village and spread amongst athletes.

The active cases per one million of the population could not accurately quantify the number of infected athletes as the correlation between the active cases and the athletes is quite low. It could only be roughly estimated as athletes could not be represented by the general population of their country. The vaccination rate of the athletes is not taken into account. With the athletes less prone to be infected by the virus after vaccination, the number of infected athletes might be even lower than the number predicted. Since the playbook and IOC did not disclose specifics for the infrastructure of their facilities and the actions that will be taken to execute these measures, the implications made under the ambiguous information given cannot be definitive.

We estimate there are approximately 32 infected athletes in the Tokyo Olympics 2020, which is close to the reported 28 athletes [17]. As our prediction only takes into account the measures inside the Olympic Village, only the infected athletes who live inside will be considered. Furthermore, our prediction was the probable number of infected athletes entering the Olympic Village after testing. The number of athletes reported might have been infected after entering the Village by other athletes or service providers, so the number of infected athletes reported would be higher than our estimated value.

Overall, if there are positive cases amongst the athletes inside the Olympic Village, it can suggest that the policies settled in the playbook for Olympic Village are insufficient and the intended COVID-19 bubble is broken. The intent of the COVID-19 bubble was to create a COVID-19 free environment. With the bubble broken, the risk of the athletes contaminating each other is created and it would no longer be a COVID-19 free environment.

The COVID-19 bubble is undeniably broken with not only one but multiple positive cases among athletes before and during the Games [17]. The spread within the Olympic Village between athletes could also be a possibility as many athletes infected are from the same country and some even the same sport. This includes two South African football players, three beach volleyball players, four Czech Republican players, and five Dutch players in particular [17]. This is a testament to how the prevention measures in place from the playbook failed to truly keep out the virus to create a safe environment for the competing athletes.

\section{DATA AVAILABILITY STATEMENT}

Publicly available datasets were analyzed in this study. This data can be found here: https://www.eurosport.com/olympics/ athletes/country/all.

\section{AUTHOR CONTRIBUTIONS}

VY, LW, BC and ZD: conceived the study, designed statistical and modeling methods, conducted analyses, interpreted results, wrote and revised the article; MX: interpreted results, and revised the article.

\section{ACKNOWLEDGMENTS}

We acknowledge the financial support from the Health and Medical Research Fund, Food and Health Bureau, Government of the Hong Kong Special Administrative Region (grant no. COVID190118), and Seed Fund for Basic Research for New Staff of the University of Hong Kong (202009185062). National Natural Science Foundation of China (grant no. 72104208). 


\section{REFERENCES}

1. ArcGIS. COVID-19 Dashboard by the Center for Systems Science and Engineering (CSSE) at Johns Hopkins University (JHU) (2020). Available at: https://gisanddata.maps.arcgis.com/apps/opsdashboard/index.html\#/ bda7594740fd40299423467b48e9ecf6 (Accessed September 28, 2020).

2. Reuters. Olympics-Tokyo's Delayed and Disrupted 2020 Games (2021). Available at: https://www.reuters.com/article/us-olympics-2020-timelineidCAKCN2EE1IN (Accessed July 21, 2021).

3. Illmer A. Tokyo Olympics: Why Doesn't Japan Cancel the Games? BBC (2021). Available at: https://www.bbc.com/news/world-asia-57097853 (Accessed July 21, 2021).

4. Wade S. Official Costs of Tokyo Olympics up by $22 \%$ to $\$ 15.4$ Billion. Associated Press (2020). Available at: https://apnews.com/article/tokyo-coronaviruspandemic-2020-tokyo-olympics-japan-olympic-games3c46bce81928865d9aae0832b5ddd9e3 (Accessed July 21, 2021).

5. Toyo Keizai. Coronavirus Disease (COVID-19) Situation Report in Japan (2021). Available at: https://toyokeizai.net/sp/visual/tko/covid19/en.html (Accessed July 21, 2021).

6. Ryall J. Tokyo Olympics: "Japanese Only" Signs Spark Outrage as Sponsors Count Cost of Spectator Ban and Covid-19 State of Emergency. South China Morning Post (2021). Available at: https://www.scmp.com/week-asia/healthenvironment/article/3140792/tokyo-olympics-japanese-only-signsspark-outrage (Accessed July 21, 2021).

7. IOC. Third Version of Tokyo 2020 Playbooks Published (2021). Available at: https://olympics.com/ioc/tokyo-2020-playbooks (Accessed July 21, 2021).

8. Tokyo. Summer Olympics Participating Countries: 206 NOCs Nations List (2020). Available at: https://www.whereig.com/olympics/summer-olympicsparticipating-countries.html (Accessed August 2, 2021).

9. Keith M. Here's what Medical Experts Say Could Improve COVID-19 Safety Measures at the Tokyo Olympics. Insider (2021). Available at: https://www. insider.com/covid-19-safety-measures-at-the-tokyo-olympics-2021-7 (Accessed July 23, 2021).

10. Worldometers. COVID Live Update: 201,777,519 Cases and 4,281,876 Deaths from the Coronavirus - Worldometer (2021). Available at: https://www. worldometers.info/coronavirus/(Accessed August 6, 2021).

11. EuroSport. Athletes by Country (2021). Available at: https://www.eurosport. com/olympics/athletes/country/all (Accessed August 6, 2021).

12. Du Z, Pandey A, Bai Y, Fitzpatrick MC, Chinazzi M, Pastore Y, et al. Comparative Cost-Effectiveness of SARS-CoV-2 Testing Strategies in the USA: a Modelling Study. The Lancet Public Health (2021) 6:e184-e191. doi:10.1016/s2468-2667(21)00002-5
13. Sporting News. NBA Bubble, Explained: A Complete Guide to the Rules, Teams, Schedule \& More for Orlando Games (2020). Available at: https:// www.sportingnews.com/us/nba/news/nba-bubble-rules-teams-scheduleorlando/zhap66a9hcwq1khmcex3ggabo (Accessed July 28, 2021).

14. Sparrow AK, Brosseau LM, Harrison RJ, and Osterholm MT. PlaceboControlled Trials of Covid-19 Vaccines - Why We Still Need Them. N Engl J Med (2021) 384:e2. doi:10.1056/nejmp2033538

15. Tokyo YA, Sturmer J, Sturmer J, Hoshiko E, and Kyung-Hoon K. Japan Unveils its Olympic Village, but Health Experts Remain Concerned about the Spread of Coronavirus. ABC News (2021). Available at: https://www. abc.net.au/news/2021-06-21/japan-unveils-its-coronavirus-safe-olympicvillage/100225266 (Accessed July 21, 2021).

16. Dalton CB, and Taylor J. Are COVID-19-safe Tokyo Olympics and Paralympics Really Possible? Med J Aust (2021) 215:54-5. doi:10.5694/ mja2.51159

17. Tokyo. COVID-19 Positive Case List. Tokyo Organising Committee of the Olympic and Paralympic Games (2021). Available at: https://olympics. com/tokyo-2020/en/notices/covid-19-positive-case-list (Accessed July 22, 2021).

Conflict of Interest: BC is supported by the AIR@innoHK program of the Innovation and Technology Commission of the Hong Kong SAR Government.

No potential competing interest from any commercial affiliation was reported by the authors.

The remaining author declares that the research was conducted in the absence of any commercial or financial relationships that could be construed as a potential conflict of interest.

Publisher's Note: All claims expressed in this article are solely those of the authors and do not necessarily represent those of their affiliated organizations, or those of the publisher, the editors, and the reviewers. Any product that may be evaluated in this article, or claim that may be made by its manufacturer, is not guaranteed or endorsed by the publisher.

Copyright (c) 2021 Vico Lau, Xu, Wang, Cowling and Du. This is an open-access article distributed under the terms of the Creative Commons Attribution License (CC $B Y)$. The use, distribution or reproduction in other forums is permitted, provided the original author(s) and the copyright owner(s) are credited and that the original publication in this journal is cited, in accordance with accepted academic practice. No use, distribution or reproduction is permitted which does not comply with these terms. 